PROCEEDINGS OF THE AMERICAN MATHEMATICAL SOCIETY

Volume 124, Number 8, August 1996

\title{
ON THE ZERO SETS OF CERTAIN ENTIRE FUNCTIONS
}

\author{
ALEXANDRE EREMENKO AND L. A. RUBEL
}

(Communicated by Albert Baernstein II)

Dedicated in gratitude to the blood donors of Champaign County

ABStract. We consider the class B of entire functions of the form

$$
f=\sum p_{j} \exp g_{j},
$$

where $p_{j}$ are polynomials and $g_{j}$ are entire functions. We prove that the zeroset of such an $f$, if infinite, cannot be contained in a ray. But for every region containing the positive ray there is an example of $f \in \mathbf{B}$ with infinite zero-set which is contained in this region.

Let $\mathbf{B}$ be Borel's class of entire functions of one complex variable that are finite sums of entire functions with only finitely many zeros (possibly none). Clearly $f \in \mathbf{B}$ if and only if

$$
f=\sum_{j=0}^{n} p_{j} \exp g_{j},
$$

where the $p_{j}$ are polynomials and the $g_{j}$ are entire functions. This class is called $B_{1}$ in [HRS].

Theorem 1. No function in $\mathbf{B}$ can have as its zero set an infinite set of positive real numbers.

Theorem 2. Given any open set $\Omega$ in the complex plane that contains the positive real axis, there is a function $f$ in $\mathbf{B}$ whose zero set is an infinite subset of $\Omega$.

Proof of Theorem 1. We will use H. Cartan's theory of holomorphic curves [C, L]. An $n+1$-vector of entire functions $\left(f_{0}, \ldots, f_{n}\right)$ without zeros common to all $f_{j}$ defines a holomorphic curve $F$ which is a holomorphic map of the complex plane $\mathbf{C}$ into the complex projective space $\mathbf{P}^{n}$. The characteristic $T(r, F)$ is defined in the following way:

$$
T(r, F)=\frac{1}{2 \pi} \int_{0}^{2 \pi} \max \left(\log \left|f_{0}\right|, \ldots, \log \left|f_{n}\right|\right)\left(r e^{i \theta}\right) d \theta .
$$

For any vector $\mathbf{a}=\left(a_{0}, \ldots a_{n}\right) \in \mathbf{C}^{n+1} \backslash\{0\}$ define

$$
N(r, \mathbf{a}, F)=\frac{1}{2 \pi} \int_{0}^{2 \pi} \log \left|a_{0} f_{0}+\ldots+a_{n} f_{n}\right|\left(r e^{i \theta}\right) d \theta .
$$

Received by the editors November 14, 1994 and, in revised form, February 7, 1995.

1991 Mathematics Subject Classification. Primary 30D15.

Research supported in part by the National Security Agency.

(C)1996 American Mathematical Society 
Such a vector a defines a hyperplane in $\mathbf{P}^{n}$ by the equation $a_{0} x_{0}+\ldots+a_{n} x_{n}=0$. If we denote by $n(r, \mathbf{a}, F)$ the number of preimages of this hyperplane under $F$ which are contained in the disk $\{z:|z| \leq r\}$, then by the Jensen formula

$$
N(r, \mathbf{a}, F)=\int_{0}^{r}\{n(t, \mathbf{a}, F)-n(0, \mathbf{a}, f)\} \frac{d t}{t}+n(0, \mathbf{a}, F) \log r+\text { const. }
$$

If $n=1$, the Cartan characteristic $T(r, F)$ coincides (up to an additive constant) with the usual Nevanlinna characteristic of the meromorphic function $f_{1} / f_{0}$. We will use the Second Main Theorem of Cartan, which (in a simplified form) states the following: Let $\mathbf{a}_{1}, \ldots, \mathbf{a}_{q}$ be an admissible system of vectors; that is, any $n+1$ of them are linearly independent. If the components $f_{0}, \ldots, f_{n}$ of a curve $F$ are linearly independent, then

$$
\sum_{j=1}^{q} N\left(r, \mathbf{a}_{j}, F\right) \geq(q-n-1+o(1)) T(r, F), \quad r \in \mathbf{R}^{+} \backslash E,
$$

where $E$ is an exceptional set of finite length.

The following theorem due to E. Borel (see, for example [L, p. 186]) is a simple corollary of the Second Main Theorem of Cartan. Let $f_{j}=p_{j} \exp g_{j}$, where $p_{j} \neq 0$ are polynomials and $g_{j}$ are entire functions. If $\left\{f_{0}, \ldots, f_{n}\right\}$ are linearly dependent, then there are two functions $\exp g_{j}$ and $\exp g_{k}$, which are proportional (with constant coefficients).

It follows from Borel's theorem that every function of the class $\mathbf{B}$ can be written in reduced form, namely the functions $f_{j}=p_{j} \exp g_{j}$ in (1) are linearly independent. Furthermore in the proof of Theorem 1 we may assume without loss of generality that $f$ is transcendental, the polynomials $p_{j}$ have no zeros common to all $p_{j}$ and that $g_{0}=0$.

With these assumptions we introduce the holomorphic curve $F$ with coordinates $f_{j}=p_{j} \exp g_{j}, 0 \leq j \leq n$, and show first that

$$
r=O(T(r, F)), \quad r \rightarrow \infty .
$$

Because $f$ in (1) is assumed to be transcendental, at least one of $g_{j}$ is not constant. Assume that $g_{n} \neq$ const. Then by the definition of characteristic and by our assumption that $g_{0}=0$ we have

$$
\begin{aligned}
2 \pi T(r, F) & \geq \int_{0}^{2 \pi} \max \left\{\log \left|f_{0}\right|, \log \left|f_{n}\right|\right\} d \theta \\
& \geq \int_{0}^{2 \pi} \max \left\{0, \operatorname{Re} g_{n}\right\} d \theta+O(\log r) \geq c r+O(\log r)
\end{aligned}
$$

for some $c>0$, which proves (3).

We need the following estimate

$$
T(r, f) \leq T(r, F)+O(\log r), \quad r \rightarrow \infty .
$$


To prove this we use first the inequality $\log |a+b| \leq \max \{\log |a|, \log |b|\}+\log 2$ and then our assumption that $g_{0}=0\left(\operatorname{so} \log \left|f_{0}\right|=\log \left|p_{0}\right|=O(\log r)\right)$ :

$$
\begin{aligned}
2 \pi T(r, f) & =\int_{0}^{2 \pi} \log ^{+}|f| d \theta \\
& \leq \int_{0}^{2 \pi} \max \left\{\log \left|f_{0}\right|, \ldots, \log \left|f_{n}\right|\right\}^{+} d \theta+O(1) \\
& =\int_{0}^{2 \pi} \max \left\{0, \log \left|f_{1}\right|, \ldots, \log \left|f_{n}\right|\right\} d \theta+O(\log r) \\
& \leq \int_{0}^{2 \pi} \max \left\{\log \left|f_{0}\right|, \ldots, \log \left|f_{n}\right|\right\} d \theta+O(\log r) \\
& =2 \pi T(r, F)+O(\log r) .
\end{aligned}
$$

Now we apply the Second Main Theorem of Cartan with $q=n+2$, and the following vectors: $\mathbf{a}_{j}$ for $1 \leq j \leq n+1$ is the $j$-th row of the $(n+1) \times(n+1)$ unit matrix and $\mathbf{a}_{n+2}=(1, \ldots, 1)$ is the row of 1 's. Then we have $N\left(r, \mathbf{a}_{j}, F\right)=O(\log r), r \rightarrow \infty$, and $N\left(r, \mathbf{a}_{n+2}, F\right)=N(r, 0, f)$, the usual Nevanlinna counting function of zeros of the entire function $f$. From (2) it follows that

$$
N(r, 0, f) \geq(1+o(1)) T(r, F), \quad r \in \mathbf{R}^{+} \backslash E .
$$

Combined with (4) this implies

$$
N(r, 0, f) \sim T(r, f), \quad r \rightarrow \infty, r \in \mathbf{R}^{+} \backslash E .
$$

In particular, this asymptotic equality combined with (3) implies that the genus of $f$ is at least 1 (maybe infinite).

Finally we use the following result of A. Edrei and W. Fuchs $[\mathrm{EF}]$ and J. Miles $[\mathrm{M}]$ : If $f$ is an entire function of genus at least 1 , with positive zeros, then there is a set $E_{1}$ of zero logarithmic density and a constant $\epsilon>0$ such that

$$
N(r, 0, f) \leq(1-\epsilon) T(r, f), \quad r \in \mathbf{R} \backslash E_{1} .
$$

Since this estimate is incompatible with (6), Theorem 1 must hold.

Proof of Theorem 2. By taking a smaller region if necessary (but still including the positive real axis), we may assume that $\Omega$ is connected and simply connected, and is bounded by a single smooth simple curve $\gamma:[-1,1] \rightarrow \mathbf{C}$ such that $\gamma(t) \rightarrow \infty$ as $t \rightarrow \pm 1$ and $\gamma$ intersects the real axis once (this intersection happens on the negative ray). The complement $T$ of $\Omega$ is an Arakelyan set, i.e. $\Omega$ is connected and locally connected at $\infty$ (see [GAI]). Using the Arakelyan approximation theorem [GAI] we find a non-constant entire function $g$ with the property $|g(z)-1 / 2|<1 / 4, z \in T$. Thus $g^{-1}(\mathbf{Z}) \subset \Omega$ and $f(z)=\exp [2 \pi i g(z)]-1$ gives the required example.

\section{REFERENCES}

[C] H. Cartan, Sur les zéros des combinations linéaires de p fonctions holomorphes données, Mathematica (Cluj), 7 (1933), 5-31.

[EF] A. Edrei and W. H. J. Fuchs, On the growth of meromorphic functions with several deficient values, TAMS, 93 (1959), 292-328. MR 22:770

[GAI] D. Gaier, Lectures on Complex Approximation, Birkhäuser, Boston-Basel-Stuttgart, 1987. MR 88i:30059b 
[HRS] C. Ward Henson, Lee A. Rubel and Michael F. Singer, Algebraic properties of the ring of general exponential polynomials, Complex Variables 13 (1989), 1-20. MR 90m:32006

[L] S. Lang, Introduction to Complex Hyperbolic Spaces, Springer, NY, 1987. MR 88f:32065

$[\mathrm{M}] \mathrm{J}$. Miles, On entire functions of infinite order with radially distributed zeros, Pacific. J. Math., 81 (1979), 131-157. MR 80i:30046

Department of Mathematics, Purdue University, West Lafayette, Indiana 47907

E-mail address: eremenko@math.purdue.edu

Department of Mathematics, University of Illinois, 1409 West Green Street, UrBANA, ILLINOIS 61801 